\title{
Identification of distinct SET/TAF-I $\beta$ domains required for core histone binding and quantitative characterisation of the interaction
} Zoe Karetsou ${ }^{1}$, Anastasia Emmanouilidou1 ${ }^{1}$, Ioannis Sanidas², Stamatis Liokatis ${ }^{1}$, Eleni Nikolakaki ${ }^{2}$, Anastasia S Politou* ${ }^{*}+1,3$ and Thomais Papamarcaki* $+1,3$

\author{
Address: ${ }^{1}$ Laboratory of Biological Chemistry, Medical School, University of Ioannina, 45110 Ioannina, Greece, ${ }^{2}$ Laboratory of Biochemistry, \\ Department of Chemistry, Aristotle University of Thessaloniki, 54124 Thessaloniki, Greece and ${ }^{3}$ Foundation for Research and Technology-Hellas/ \\ Biomedical Research Institute, 45110 Ioannina, Greece \\ Email: Zoe Karetsou - zkarets@cc.uoi.gr; Anastasia Emmanouilidou - aemmanou@cc.uoi.gr; Ioannis Sanidas - gsanid@chem.auth.gr; \\ Stamatis Liokatis - me00537@cc.uoi.gr; Eleni Nikolakaki - nikol@chem.auth.gr; Anastasia S Politou* - apolitou@cc.uoi.gr; \\ Thomais Papamarcaki* - thpapama@uoi.gr \\ * Corresponding authors †Equal contributors
}

Published: 9 April 2009

BMC Biochemistry 2009, 10:10 doi:10.1186/1471-2091-10-10

This article is available from: http://www.biomedcentral.com//47I-209///0/10

(C) 2009 Karetsou et al; licensee BioMed Central Ltd.

This is an Open Access article distributed under the terms of the Creative Commons Attribution License (http://creativecommons.org/licenses/by/2.0), which permits unrestricted use, distribution, and reproduction in any medium, provided the original work is properly cited.

\begin{abstract}
Background: The assembly of nucleosomes to higher-order chromatin structures is finely tuned by the relative affinities of histones for chaperones and nucleosomal binding sites. The myeloid leukaemia protein SET/TAF-I $\beta$ belongs to the NAPI family of histone chaperones and participates in several chromatin-based mechanisms, such as chromatin assembly, nucleosome reorganisation and transcriptional activation. To better understand the histone chaperone function of SET/TAF$I \beta$, we designed several SET/TAF-I $\beta$ truncations, examined their structural integrity by circular Dichroism and assessed qualitatively and quantitatively the histone binding properties of wild-type protein and mutant forms using GST-pull down experiments and fluorescence spectroscopy-based binding assays.
\end{abstract}

Results: Wild type SET/TAF-I $\beta$ binds to histones $\mathrm{H} 2 \mathrm{~B}$ and $\mathrm{H} 3$ with $\mathrm{K}_{\mathrm{d}}$ values of 2.87 and $0.15 \mu \mathrm{M}$, respectively. The preferential binding of SET/TAF-I $\beta$ to histone $\mathrm{H} 3$ is mediated by its central region and the globular part of $\mathrm{H} 3$. On the contrary, the acidic $\mathrm{C}$-terminal tail and the amino-terminal dimerisation domain of SET/TAF-I $\beta$, as well as the $\mathrm{H} 3$ amino-terminal tail, are dispensable for this interaction.

Conclusion: This type of analysis allowed us to assess the relative affinities of SET/TAF-I $\beta$ for different histones and identify the domains of the protein required for effective histone recognition. Our findings are consistent with recent structural studies of SET/TAF-I $\beta$ and can be valuable to understand the role of SET/TAF-I $\beta$ in chromatin function.

\section{Background}

The genomes of eukaryotic cells are organized as chromatin, a highly dynamic complex between DNA and histones. The fundamental repeating unit in chromatin is the nucleosome core particle (NCP), which is composed of 147 bp of DNA wrapped around an octameric core containing two copies of each of the four histones H2A, H2B, $\mathrm{H} 3$, and $\mathrm{H} 4$ [1]. Ultimately, long arrays of nucleosomes 
are further compacted into higher-order structures that form functional chromatin domains. The packaging of DNA into chromatin ensures genome stability, but at the same time creates a barrier for the access of cellular factors to underlying DNA sequences, thus influencing gene expression, DNA replication, recombination and repair [2]. The major mechanisms which modulate chromatin structure include ATP-dependent nucleosome remodeling complexes, covalent modification of histones, histone variant exchange and histone chaperones [3]. ATPdependent remodeling complexes use the energy of ATP hydrolysis to regulate chromatin fluidity, leading to either activation or repression of transcription $[4,5]$. Recent studies have revealed a large number of post-translational modifications that lie either in the flexible histone tails or in the histone globular domain, which are correlated with distinct functional states of chromatin [6-8]. In concert to these machineries, non-histone proteins and histone chaperones regulate nucleosome dynamics by influencing the binding affinity of histones for DNA [9].

Histone chaperones are key players in the organisation of chromatin domains [9-11]. They shield the positive charge of histones and safeguard them from improper electrostatic interactions with DNA or other proteins which could be harmful for the cell. Consequently, they mediate chromatin assembly/disassembly, histone storage and coordinate the exchange/deposition of histones onto DNA during replication. The best characterised chaperones are NAP1 $[12,13]$, chromatin assembly factor 1 (CAF1) [14], N1/N2 [15], nucleoplasmin [16], antisilencing factor (ASF1) [17] and HIRA [18]. Despite the fact that all chaperones bind to histones, they seem to mediate distinct protein-protein interactions in vivo and their histone partners and binding affinities vary considerably.

The myeloid leukaemia protein SET/TAF- $1 \beta$ studied here, also known as $\mathrm{I}_{2} \mathrm{PP} 2 \mathrm{~A}$, and INHAT, belongs to the NAP1 family of histone chaperones. It was originally identified as a translocated gene in acute undifferentiated leukemia [19-21] and is widely expressed in human and mouse tissues [22]. The analysis of SET/TAF-I $\beta$ functions is challenging, because it is involved in a broad range of cellular mechanisms and it remains unclear how it switches between these different functions. SET/TAF-I $\beta$ is a potent inhibitor of phosphatase 2A (PP2A), and interacts with several proteins involved in the regulation of cell cycle, such as p21waf1 [23], cyclin E-CDK2 [23], B- cyclins [24] and p35nck5a [25]. Furthermore, SET/TAF-I $\beta$ has been identified as a key factor of the cytotoxic T lymphocytes (CTL)-induced cell death, suggesting its role in the regulation of apoptosis [26]. Besides its involvement in the control of cell cycle and apoptosis, increasing number of reports describe the chromatin-related properties of SET/
TAF-I $\beta$. It stimulates DNA replication and transcription of the adenovirus genome [27-30] and induces chromatin decondensation [31]. Other studies have shown that SET/ TAF-I $\beta$ binds to nucleosomal histones [32-34] and inhibits histone acetylation by masking histone tails as a component of the INHAT complex [35]. In addition, we have previously reported the interaction of SET/TAF-I $\beta$ with the chromatin remodeling protein prothymosin $\alpha$ [36] and the transcription coactivator CREB-binding protein (CBP) [37]. Consistent with the above studies, a DNA-microarray based analysis revealed that SET/TAF-I $\beta$ stimulates the transcription of a sub-set of genes [38]. Interestingly, the transcription of the endogenous genes which were upregulated by SET/TAF-I $\beta$ was found to be additively stimulated by histone acetylation. Recently, Ichijo et al [39] showed that Set/TAF-I $\beta$ interacts with the activated glucocorticoid receptor and acts as a ligand-activated GRresponsive transcriptional repressor, which further suggests the involvement of this protein in transcriptional regulation.

Inspection of the aminoacid sequence of SET/TAF-I $\beta$ reveals three interesting structural features, which are important determinants of its ligand binding activity and, ultimately, of its functional specificity. First, a motif located in the $\mathrm{N}$-terminal region (amino acids 1-76) predicted to form a coiled-coil structure, which is believed to mediate the dimerisation of the protein [40]. Dimerisation is a conserved feature of the NAP1 family of histone chaperones and it was found to be necessary for the chromatin remodeling activity of SET/TAF-I $\beta$ [40]. Second, the central NAP domain (amino acids 80-225), which is highly conserved among the NAP1 protein family members and is thought to mediate histone fold-specific binding $[12,13]$. Third, a highly acidic polyglutamic track located in the C-terminal tail of SET/TAF-I $\beta$ (amino acids 226-277). This region consists of 42 glutamic and aspartic residues occasionally interspersed by glycine residues and seems to adopt an intrinsically disordered structure that provides structural plasticity, according to recent CD and X-Ray data on yeast NAP1 $[12,41]$. In vitro experiments suggested that the acidic tail might be implicated in the chaperone activity of SET/TAF-I $\beta$, targeting the basic $\mathrm{N}$-terminal tails of histones [31,42]. However, it is still unclear which domains of the protein are required for effective histone recognition. In addition, the relative affinity of SET/TAF-I $\beta$ for the various histones remains unknown. This information can be very valuable to understand the role of SET/TAF-I $\beta$ as a histone chaperone, since histone binding activity is the common feature of this class of proteins, through which they are involved in a wide range of mechanisms, besides nucleosome assembly/disassembly, such as histone variant exchange, histone modification regulation, histone shuttling and storage and transient histone overload buffering [43,44]. 
Moreover, it has been recently shown that the assembly of nucleosomes to the level of chromatin is governed by affinity differences of histones between chaperones and binding sites on subnucleosomal complexes $[41,45]$.

Here, we focused on the histone-binding properties of SET/TAF-I $\beta$. We performed GST-pull down analysis with wild type SET/TAF-I $\beta$ and all five histones and fluorescence spectroscopy-based quantitative binding assays with those histones that appeared to interact with SET/ TAF-I $\beta$. This analysis allowed us to assess the relative affinity of SET/TAF-I $\beta$ for different histones. Such detailed analysis has not, to our knowledge, been carried out for this protein. Furthermore, in order to identify the regions of the protein responsible for the interaction with histones, we designed several SET/TAF-I $\beta$ truncations, examined their structural integrity by circular dichroism and assessed qualitatively and quantitatively their histonebinding properties by GST-pull down assays and fluorescence spectroscopy-based titration experiments. Collectively, our data show that SET/TAF-I $\beta$ binds preferentially to histone $\mathrm{H} 3$ through its central region. The dimerisation domain of SET/TAF-I $\beta$, as well as its C-terminal acidic tail, are not essential for this interaction.

\section{Results \\ Preferential binding of of SETITAF-I $\beta$ to histone H3}

To study the histone binding activity of SET/TAF-I $\beta$, we performed GST-pull down assays. GST-SET/TAF-I $\beta$ was immobilised on glutathione beads and challenged with $\mathrm{H} 1$ or core histones at high salt concentrations. The results revealed binding of SET/TAF-I $\beta$ to histones H2B (Fig. 1A, lane 6) and $\mathrm{H} 3$ (lane 8), while no interaction was observed with histones H1 (lane 2), H2A (lane 4) and H4 (lane 10). GST immobilized on glutathione beads was used as a negative control (not shown).

To compare quantitatively the relative affinity of SET/TAF$\mathrm{I} \beta$ for histones $\mathrm{H} 3$ and $\mathrm{H} 2 \mathrm{~B}$, we performed fluorescencebased titration experiments $[46,47]$. SET/TAF-I $\beta$ sequence contains four tryptophan residues, which could serve as intrinsic fluorescence chromophores. On the other hand, histones are poor in aromatic amino acids and completely devoid of tryptophan residues. Therefore, the binding affinity of SET/TAF-I $\beta$ to histones H3 and H2B could be monitored by fluorescence spectroscopy at an excitation wavelength that precludes a contribution to the emitted fluorescence intensity from the tyrosine residues of histones. A clear and gradual quenching of the fluorescence signal was observed, when increasing amounts of histones were added to full-length SET/TAF-I $\beta$. The fluorescence intensity change was used for a quantitative binding assay. A binding curve was obtained by monitoring the intensity at $345 \mathrm{~nm}$ of the fluorescence emitted by SET/TAF-I $\beta$ as a function of added histone concentration at constant temperature (Fig. 1B). Fitting of the fluorescence data resulted in a $K_{d}$ value of $0.15 \mu \mathrm{M}$ for the SET/TAF-I $\beta$-H3 interaction and a $K_{d}$ value of $2.87 \mu \mathrm{M}$ for the SET/TAF-I $\beta$-H2B interaction (Table 1). The qualitative and quantitative assessment of the SET/TAF-I $\beta$-histone interaction points to a clear preference of SET/TAF-1 $\beta$ for histone H3. Pull-down experiments with GST-fused SET/TAF-I $\beta$ and reconstituted $(\mathrm{H} 3 / \mathrm{H} 4)_{2}$ tetramers showed that this interaction is also efficient in the more "organized" context of the $(\mathrm{H} 3 / \mathrm{H} 4)_{2}$ tetramer (Fig. 1C). In this sense, SET/TAF-I $\beta$ behaves similarly to NAP1 from yeast, Drosophila and humans which have been shown to preferentially bind the $(\mathrm{H} 3 / \mathrm{H} 4)_{2}$ tetramer in vitro [41], although NAP family members have generally been described as $\mathrm{H} 2 \mathrm{~A} / \mathrm{H} 2 \mathrm{~B}$ chaperones. On the contrary, yeast NAP1 is capable of binding to linker histone $\mathrm{H} 1$ [48], while our data rule out such a possibility for SET/TAF-I $\beta$ (Fig. 1A, lane 2). NAP1 from yeast and humans has also been shown to facilitate the removal of H2A-H2B dimers from a folded nucleosome $[49,50]$, but in the same set of experiments, SET/TAF-I $\beta$ did not demonstrate an efficient stripping activity of the dimer [49]. This difference together with the clear preference of SET/ TAF-I $\beta$ for $\mathrm{H} 3$ binding and with the lack of interaction with linker histone H1 may account, at least in part, for distinct roles of SET/TAF-I $\beta$ in diverse mechanisms of nucleosome assembly and reorganization.

\section{Individual domains of SETITAF-I $\beta$ are sufficient for histonebinding}

Since $\mathrm{H} 3$ was identified as the preferred histone substrate of SET/TAF-I $\beta$, we proceeded to identify the regions of SET/TAF-I $\beta$ involved in $\mathrm{H} 3$ binding. Based on sequence comparisons between human SET/TAF-I $\beta$ and members of the Nucleosome Assembly Protein (NAP) family, in particular NAP-1 from yeast, whose three-dimensional structure was recently solved [12] (PDB ID: 2AYU), we designed several deletion mutants of SET/TAF-I $\beta$, shown in Fig. 2A. Our specific aim in the design of the mutants was to challenge the role in $\mathrm{H} 3$ binding of (i) the dimerisation domain, (ii) the acidic C-terminal tail and (iii) the central region of the protein. Previous structural and biochemical studies have underlined the essential role of the central region of NAP1 proteins in the interaction with histones; the $\beta$-sheet subdomain, which is part of this region, is structurally conserved in otherwise unrelated in sequence and structure histone chaperones and is believed to form a scaffold onto which histone binding elements are built [12].

Wild type SET/TAF-I $\beta$ and its mutants were expressed and purified as GST-tagged proteins and subsequently the GST moeity was removed, as indicated in the Methods section (Fig. 2B). The structural integrity of wild-type and mutant forms of SET/TAF-I $\beta$ was explored by Circular Dichroism Spectroscopy (CD). All constructs tested, with the exception of the polypeptide spanning residues 110-210 of the protein, were found well folded (Fig. 2C). The CD 

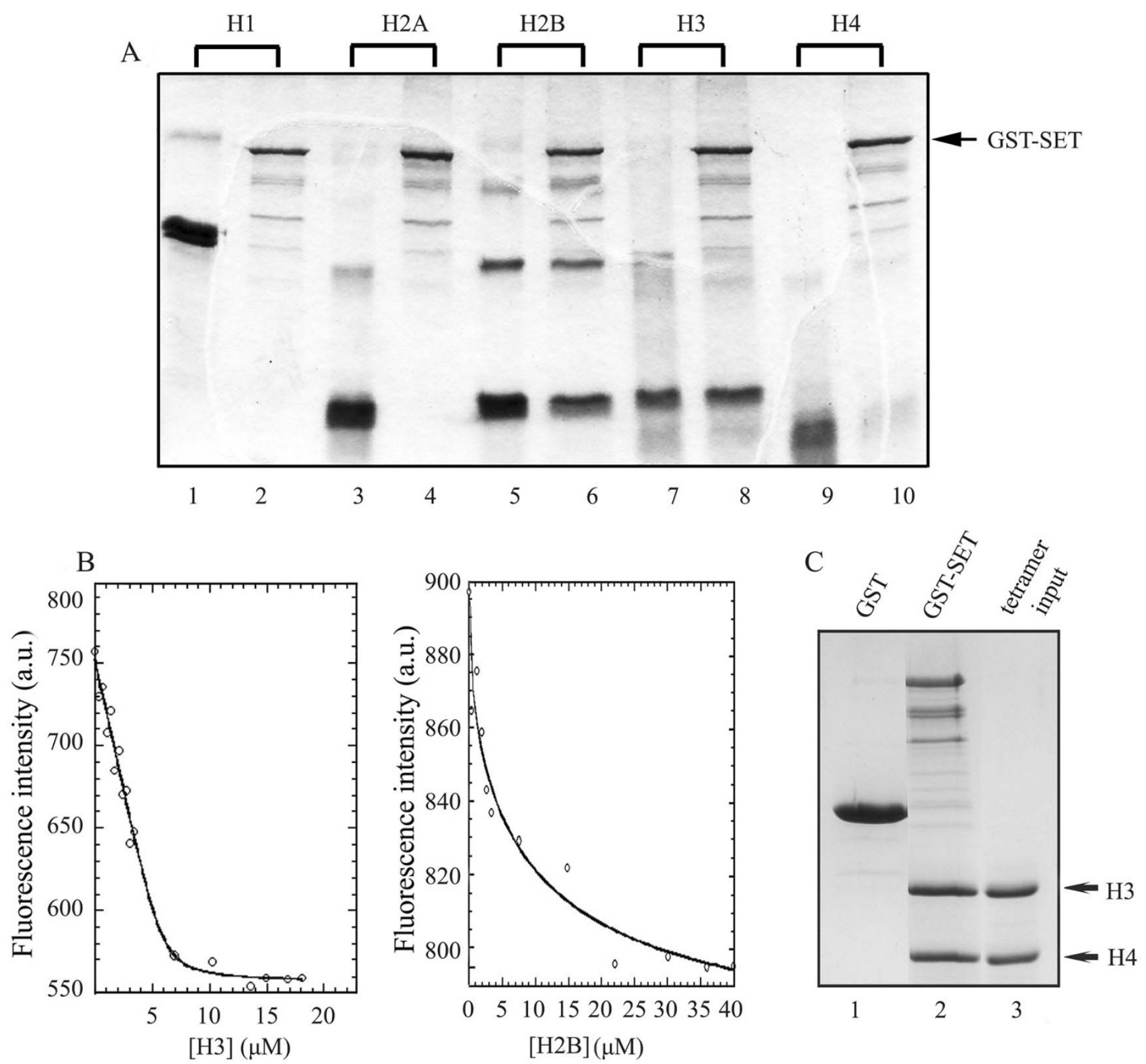

Figure I

Histone binding properties of SET/TAF-I $\beta$. (A) SET/TAF-I $\beta$ binding to histones. Purified GST-SET/TAF-I $\beta$ (2 $\mu$ g) was immobilised on glutathione-agarose beads and incubated with $4 \mu \mathrm{g}$ of $\mathrm{HI}$ (lane 2) or core histones H2A, H2B, H3, H4 (lanes 4, 6, 8, 10 , respectively) as indicated in Materials and Methods. The beads were washed and analysed by SDS-PAGE and Coomassie blue staining. Lanes I, 3, 5, 7, 9 indicate the input of the histones. (B) Fluorescence-based binding assays. SET/TAF-I $\beta$ at an initial concentration of $4 \mu \mathrm{M}$ was titrated with increasing amounts of purified calf thymus $\mathrm{H} 3$ (left panel) or $\mathrm{H} 2 \mathrm{~B}$ (right panel) stock solutions in PBS buffer. SET/TAF-I $\beta$ binding to histones $\mathrm{H} 3$ and $\mathrm{H} 2 \mathrm{~B}$ was followed by monitoring the intensity of the fluorescence emitted at $345 \mathrm{~nm}$ after excitation at $295 \mathrm{~nm}$. The solid line represents the binding curve derived by non-linear regression analysis of the fluorescence values corrected for dilution and for buffer contribution. (C) SET/TAF-I $\beta$ binding to (H3/H4) 2 tetramers. GST (lane I) and GST-SET/TAF-I $\beta$ (lane 2) were immobilised on glutathione-agarose beads and incubated with reconstituted $(\mathrm{H} 3 / \mathrm{H} 4)_{2}$ tetramers (lane 3$)$ as indicated in Materials and Methods. The beads were washed and bound proteins were analysed by SDS-PAGE and Coomassie blue staining. 
Table I: Relative affinities of SET-TAFI- $\beta$ and its deletion mutants for $\mathrm{H} 3$

\begin{tabular}{lcc}
\hline SET/TAF-I $\beta$ construct & $\mathbf{K}_{\mathbf{d}}$ & Pull-down results \\
\hline SET I-277 & $0.15 \pm 0.20$ & + \\
SET I-210 & $0.13 \pm 0.10$ & + \\
SET 76-210 & $0.14 \pm 0.08$ & + \\
SET 76-185 & $0.10 \pm 0.08$ & + \\
SET I 10-277 & $0.16 \pm 0.12$ & + \\
SET I 10-210 & n.d. & - \\
\hline
\end{tabular}

Binding of the various SET/TAF-I $\beta$ deletion mutants with histone $\mathrm{H} 3$ was followed by monitoring the intensity of the fluorescence emitted by the tryptophan residues of SET/TAF-I $\beta$ polypeptides upon addition of increasing amounts of calf thymus histone $\mathrm{H} 3$. The dissociation constant $\left(K_{d}\right)$ for the complexes formed was estimated by non-linear regression analysis (KaleidaGraph, Synergy Software), as indicated in Methods.

spectrum of full-length SET/TAF-I $\beta$ is indicative of a predominantly $\alpha$-helical protein. The proportion of the two main types of secondary structure varies significantly between the different mutants, depending on whether the $\mathrm{N}$-terminal $\alpha$-helical part or the C-terminal disordered region is included in each construct. It should be noted at this point that the C-terminal acidic stretch, the only part of the protein expected to be completely disordered in solution, was not analysed by CD due to technical problems during its purification.

In order to test the binding activity of SET/TAF-I $\beta$ fragments for histone H3, we first carried out series of GSTpull down experiments with all GST-tagged mutants and H3. As shown in Figure 3A, all SET/TAF-I $\beta$ fragments had the ability to interact with $\mathrm{H} 3$, with the exception of the polypeptide SET/TAF-I $\beta$ 110-210, which was found misfolded and aggregated. These results suggest that the binding site of SET/TAF-I $\beta$ for $\mathrm{H} 3$ resides mainly within its central region, despite the fact that the protein possesses a $\mathrm{C}$-terminal region of pronounced negative charge. Moreover, it appears that dimerisation of SET/TAF-I $\beta$ is not essential for the interaction, since mutants lacking the complete $\mathrm{N}$-terminal dimerisation domain (residues 1 76) do not fail to bind H3 (Fig. 3A, lanes 6, 9, 10).

To assess the relative affinities of the isolated SET/TAF-I $\beta$ deletion mutants for H3 (Fig. 2B), we resorted again to fluorescence-based titration experiments. All SET/TAF-I $\beta$ mutants have one or more tryptophan residues in their sequence which could function as intrinsic fluorescence chromophores. The results are consistent with the pull down assays, supporting further the importance of the central region of SET in its interaction with H3. They show that, in general, shorter constructs centered around the central region of SET/TAF-I $\beta$ (amino acids 76-185) and lacking either the N-terminal dimerisation domain and/ or the acidic C-terminal tail are sufficient for $\mathrm{H} 3$ binding and their affinities for $\mathrm{H} 3$ are comparable to that of the full length protein (summarized in Table 1). The homologous region of yeast NAP1 has been shown to be crucial for the interaction with histones and has been termed "protein interaction domain" [12]. Besides the length of the mutants able to bind $\mathrm{H} 3$, the values of $\mathrm{K}_{\mathrm{d}}$ 's of most complexes and the stoichiometry deduced from the binding curves, clearly indicate that neither dimerisation nor the acidic tail-domain of SET/TAF-I $\beta$ are absolutely necessary for the interaction (for example, compare the $\mathrm{K}_{\mathrm{d}}$ 's of the complexes H3-SET/TAF-I $\beta$ (1-277) and H3-SET/TAFI $\beta$ (76-210) (Table 1). Our data are in agreement with previous observations on the 1:1 stoichiometry for yeast NAP1/H3 interaction [41].

We next attempted to identify the region of histone H3, which is involved in its binding with SET/TAF-I $\beta$. To this end, GST-fused recombinant $\mathrm{H} 3$ tail was tested for its abilitity to bind wild type and SET/TAF-I $\beta$ (76-185) in pull down assays. Figure $3 \mathrm{~B}$ shows that no binding to the amino terminal tail of $\mathrm{H} 3$ was detected. In contrast, binding of both proteins to the globular domain of $\mathrm{H} 3$ was observed (Fig. 3C, lanes 1,2). These results suggest that the $\mathrm{H} 3 \mathrm{~N}$-terminal tail is not required for its interaction with SET/TAF-I $\beta$. On the contrary, they indicate that binding determinants of the SET/TAF-I $/ \mathrm{H} 3$ interaction lie within the globular domain of H3. This finding contradicts a previous study showing direct binding of SET/TAF$\mathrm{I} \beta$ to the $\mathrm{H} 3 \mathrm{~N}$-terminal tail, which is disrupted when the tail is modified [34]. Discrepancies between the two studies might result from differences in the assays and the H3 fragments used. We tested full-length, native histone H3 with wild type SET/TAF-I $\beta$ and its deletion mutants in our binding assays (Figs. 1A, 1B, 3A). We also used recombinant forms of full length $\mathrm{H} 3$ (Fig. 3C, lanes 4, 5), the globular part of $\mathrm{H} 3$ and its $\mathrm{N}$-terminal tail (H3 residues 146). On the contrary, in the above study, SET/TAF-I $\beta$ binding to $\mathrm{H} 3$ was tested using short synthetic peptides corresponding to residues $1-16$ of $\mathrm{H} 3$, immobilized on columns [34]. Our data clearly show that the binding of SET/TAF-I $\beta$ to H3 occurs with native H3 (Figs. 1A, 1B, 3A), which is heavily modified (S. Liokatis and A.S. Politou, unpublished results) and with recombinant forms of intact $\mathrm{H} 3$ and of its core region (Fig. 3C), which are devoid of any post-translational modifications. On the other hand, the binding is abolished when unmodified $\mathrm{H} 3$ tail is used in the pull-down assays (Fig. 3B). Therefore, neither the amino-terminal tail nor any modifications (either in the core or in the tail) of $\mathrm{H} 3$ seem to play an essential role in its interaction with SET/TAF-I $\beta$.

\section{Discussion}

The histone binding studies described in this work were made with wild-type and SET/TAF-I $\beta$ deletion mutants. These mutants were designed on the basis of its sequence similarity to NAP-1 from yeast, whose three-dimensional 
A



B



C

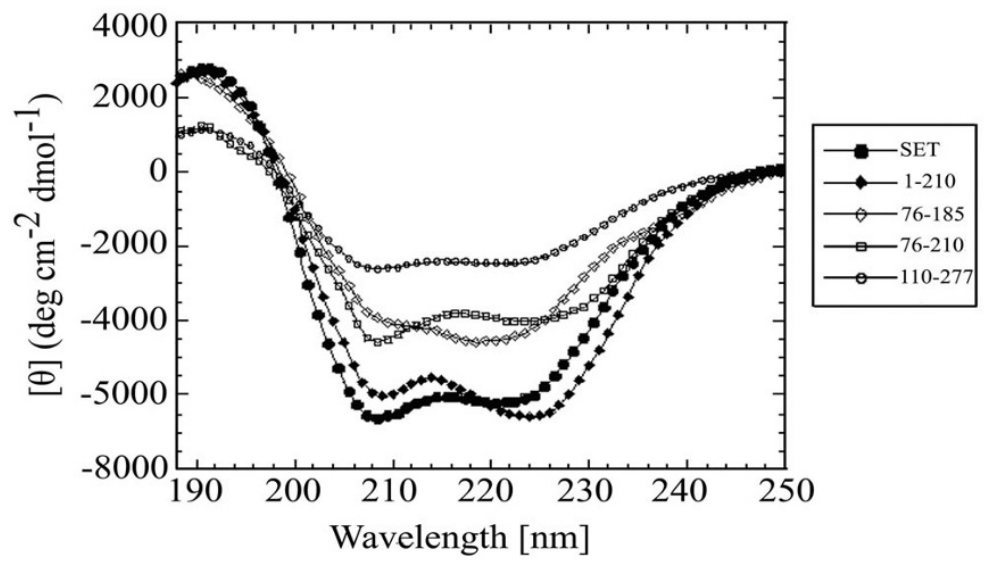

\section{Figure 2}

Design and structural integrity of SET/TAF-I $\beta$ deletion constructs. (A) Aminoacid sequence and secondary structure elements of SET/TAF-I $\beta$ (PBD code 2E250) and its deletion mutants; SET/TAF-I $\beta$ (I-2I0) (yellow), SET/TAF-I $\beta$ (76-2I0) (red), SET/TAF-I $\beta$ (76-185) (blue), SET/TAF-I $\beta$ (I I0-277) (orange), SET/TAF-I $\beta$ (I I0-2 I0) (green)(insert). Residues in the acidic stretch are shown in grey. (B) Wild type SET/TAF-I $\beta$ and its deletion mutants were expressed and purified as GST-tagged proteins and subsequently the GST moiety was removed, as indicated in the Methods section. The proteins were analysed by SDSPAGE and Coomassie blue staining. (C) Circular dichroism spectra of wild type SET/TAF-I $\beta$ (I-277) (filled hexagon), SET/TAFI $\beta$ (I-2I0) (filled diamond), SET/TAF-I $\beta$ I I0-277 (open hexagon), SET/TAF-I $\beta$ 76-I85 (open diamond) and SET/TAF-I $(76-$ 2 I0) (open square). Stock solutions of SET/TAF-IB polypeptides were in $50 \mathrm{mM}$ Tris- $\mathrm{HCl}$ pH 7.5, $150 \mathrm{mM} \mathrm{NaCl,} 0.5 \mathrm{mM} \mathrm{DTT}$, I mM EDTA in a concentration range of $10-75 \mu \mathrm{M}$. 


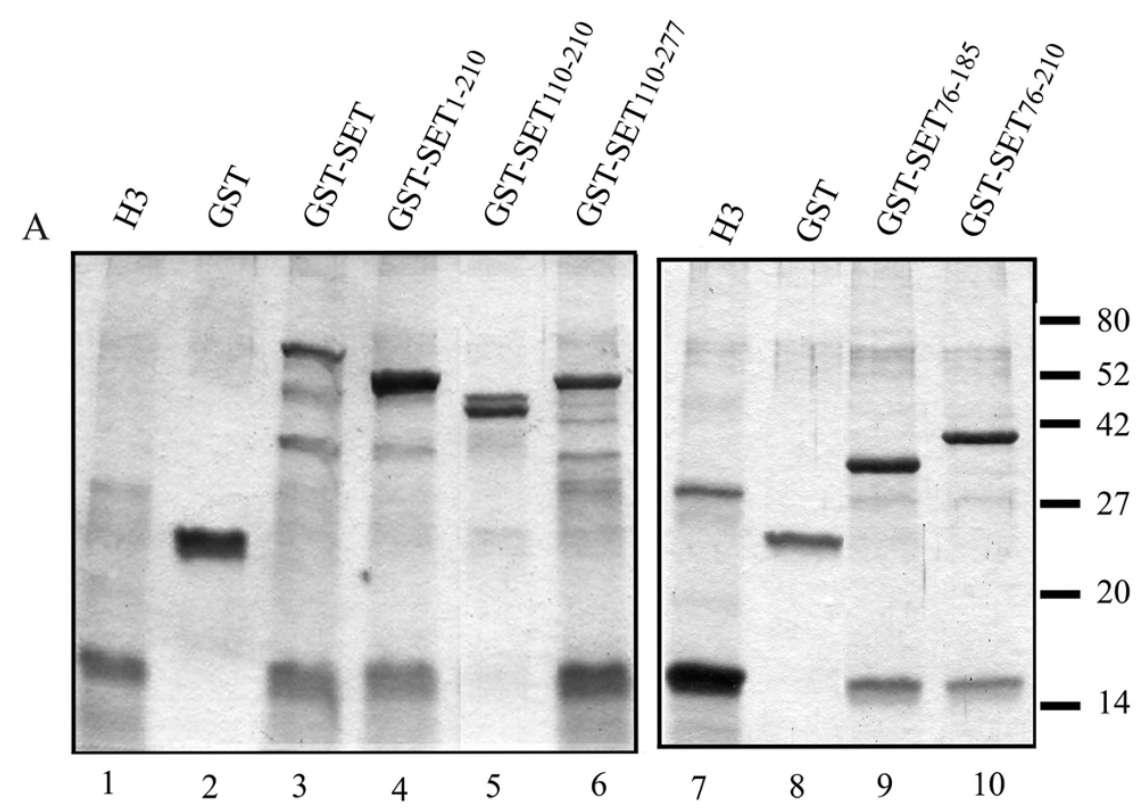

B


Figure 3

Binding of SET/TAF-I $\beta$ proteins to histone H3. (A) GST-SET/TAF-I $\beta$ (lane 3), GST-SET/TAF-I $\beta$ constructs (lanes 4,5 , 6 , $9,10)$ and purified GST (lanes 2,8$)(4 \mu \mathrm{g}$ each) were immobilised on glutathione-agarose beads and incubated with $4 \mu \mathrm{g}$ of histone H3. The beads were washed and analysed by SDS-PAGE and Coomassie blue staining. Lanes I and 7 indicate the input of histone H3. (B) GST-H3 tail (lanes I,2) or GST (lanes 3, 4) $(2 \mu \mathrm{g}$ each) were bound on glutathione-agarose beads and incubated with $4 \mu \mathrm{g}$ of SET/TAFI- $\beta$ (76-I85) (lanes I, 3) or wild type SET/TAFI- $\beta$ (lanes 2,4$)$. The beads were washed and analysed by SDS-PAGE and Coomassie blue staining. The input of SET/TAFI- $\beta$ and SET/TAFI- $\beta$ (76-185) is shown in lanes 5, 6. (C)GST (lanes 3, 6), GST-SET/TAF-I $\beta$ (lanes 2, 5), GST-SET/TAF-I $\beta$ (76-I85) (lanes I, 4) $(2 \mu \mathrm{g}$ each) bound on glutathione beads were incubated with $4 \mu \mathrm{g}$ of purified H3 globular domain (lanes I, 2, 3) or recombinant histone H3 (lanes 4, 5, 6). Bound proteins were eluted with $20 \mu \mathrm{l}$ of reduced glutathione and samples were analysed by SDS-PAGE and Coomassie blue staining.

structure was known [12], since the structure of SET/TAFI $\beta$ had not been determined yet. While this manuscript was in preparation, the crystal structure of SET/TAF-I $\beta$ was reported (PDB ID: 2E50) [51]. The crystal structure shows that SET/TAF-I $\beta$ forms a dimer that assumes a headphone like shape, with each subunit consisting only of an unstable and highly mobile $\alpha$-helix, a long backbone helix, and an $\alpha+\beta$ "earmuff domain" (amino acids 1-24, 25-78, and 79-225, respectively). The two backbone helices are not arranged in a coiled-coil fashion, but interact hydropho- 
bically in an antiparallel manner to form the dimer. The earmuff domain is attached to the concave side of the backbone helix and its lower part is highly mobile in aqueous solution. Comparison of the crystal structure of SET/TAF-I $\beta$ with that of NAP-1 revealed that the two proteins exhibit the highest similarity in the region of the earmuff domain. Inspection of the structure itself and biochemical analysis of the wild-type protein and of 18 triple mutants led to important conclusions regarding the histone and DNA binding properties of SET/TAF1 $\beta$, which provide insight into its histone chaperone function. More specifically, Muto et al. [51] have shown that: (a) SET/ TAF1 $\beta$ has a histone chaperone activity specific to $\mathrm{H} 3 / \mathrm{H} 4$, (b) its acidic C-terminal stretch is not important for H3/ H4 binding and (c) the bottom part of the earmuff domain, formed mainly by the $\beta$-structure, which is exposed to the solvent, is more likely responsible for its histone chaperone activity. No affinity measurements of the tested interactions have been reported in that work.

Our approach, based on the design of deletion mutants and functional domains of SET/TAF1 $\beta$ and on the quantitative comparison of their affinities for histones, allowed us to draw conclusions that extend and complement this study [51] by shifting the focus towards affinity differences of isolated histones for the SET/TAF1 $\beta$ chaperone.

When the domains analysed in this work are mapped onto the SET/TAF-I $\beta$ sequence it becomes clear that the central region of SET/TAF-I $\beta$, spanning residues $76-185$ in the earmuff domain, is crucial for histone binding. The sequence of this particular region is extremely conserved between human, mouse and Xenopus SET/TAF-I $\beta$ and its tertiary structure is very similar to the homologous region in yeast NAP-1 (Fig. 4). It has been recently shown that the amphipathic $\beta$-sheet formed in this region represents a structural motif common to all histone chaperones with known structures (NAP-1, nucleoplasmin, ASF1, CAF-1), despite a wide variation in sequence, structural context and overall architecture of the various chaperones $[12,13]$. These observations suggest a strong evolutionary conservation and underscore the relevance of this motif for the common function of histone chaperones with their differences accounting for distinct roles in chromatin assembly. Moreover, our data provide concrete and quantitative evidence that removal of the acidic C-terminal tail of SET/TAF-I $\beta$ does not affect its affinity for histone H3. It can be easily inferred from this observation that charge neutralization between the basic histones and acidic chaperones is dispensable for their interaction, a conclusion further supported by the complete absence of acidic stretches in certain chaperones, such as ASF1 [52]. It cannot be excluded, though, that post-translational modifications of the acidic stretch could have an effect on the interaction between chaperones and their respective histone substrates in vivo. Acidic stretches of NAP1 have been
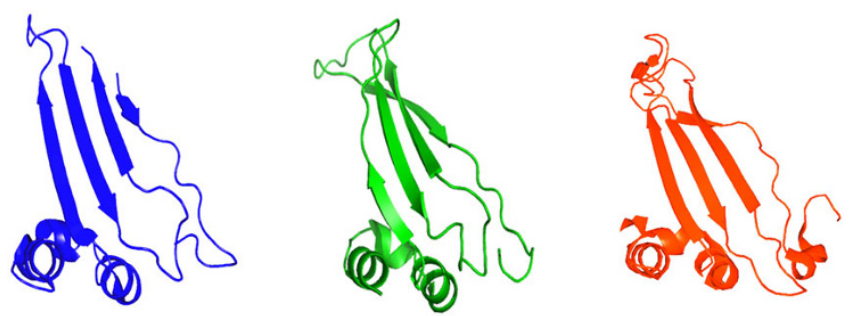

\section{Figure 4}

Mapping on the SET/TAF-I $\beta$ structure of the central region essential for $\mathbf{H} 3$ binding. Views of the central region of SET/TAF-I $\beta$ found to be essential for $\mathrm{H} 3$ binding in this work (blue), the homologous domain (named subdomain C) of yeast NAP-I (green) and the homologous domain of yeast Vps75 (red). PDB codes used are 2E50 for human SET/ TAF-I $\beta, 2 A Y U$ for yeast NAP-I and 2ZD7 for yeast Vps75.

The central $\beta$-sheet is a common structural motif found in all histone chaperones known todate. Molecular images were generated with PyMol [6I].

shown to be highly polymorphic in a variety of tissues and organisms due to various degrees of polyglutamylation [12], a modification that could significantly affect the charge and physicochemical properties of SET/TAF-I $\beta$ in a reversible manner and lead to further refinement of its interaction with histones in vivo.

Very recently three different reports on the structure and biochemistry of a new member of the histone chaperone family, Vps75 from yeast [53], revealed a remarkable structural similarity with SET/TAF-I $\beta$ and, in agreement with our observations, highlighted the importance of the central cleft for histone binding [54-56]. Comparison of the high resolution structures of Vps75 and SET/TAF-I $\beta$ clearly shows that the two proteins share, besides the overall topology, several other structural features, such as the lack of the extended N-terminal tail and of the accessory domain identified in NAP-1, which point to Vps75 as the closest known structural homologue of SET/TAF-I $\beta$ in yeast (Fig. 4). Biochemical experiments with wild-type and mutated Vsp75 show that its central cleft is important for histone binding and for its interaction with the histone acetyltransferase Rtt109. Significant functional similarities with SET/TAF-I $\beta$ include the predominantly nuclear localization, the involvement in non-canonical histone chaperone activities, the interaction with both $\mathrm{H} 2 \mathrm{~A} / \mathrm{H} 2 \mathrm{~B}$ and $\mathrm{H} 3 / \mathrm{H} 4$, the irrelevance of the $\mathrm{H} 3$ tails for this interaction and the lack of $\mathrm{H} 2 \mathrm{~A} / \mathrm{H} 2 \mathrm{~B}$ dimer stripping activity, in sharp contrast to NAP-1 which readily removes H2A/H2B dimers from assembled nucleosomes [55]. There are, however, notable differences between the two proteins, especially in the central cleft size and the intrinsic motion of this part resulting in altered charge density and surface electrostatic potential, that have been correlated with the unique function of Vps75 to interact with Rtt109 for histone acetylation, but also with likely differ- 
ent histone substrate specificities [54,56]. Evidence to this direction is provided by our quantitative binding studies, which indicate a clear preference of SET/TAF-I $\beta$ for H3 over H2B binding, unlike Vps75 that has been shown to preferentially interact with $\mathrm{H} 2 \mathrm{~A} / \mathrm{H} 2 \mathrm{~B}$ [55]. In addition, the histone chaperone activity of Vps75 has been found to be intimately linked with stimulation of $\mathrm{H} 3$ acetylation through the formation of the multifunctional Vps75Rtt109 complex that acetylates H3 and deposits H3/H4 onto DNA [54]. Affinity measurements for the interaction of H3 with wild-type and Vps75 mutants previously reported to be defective in histone binding and chaperone activity of SET/TAF-I $\beta$ [51] demonstrated that the region of Vps75 responsible for Rtt109 activation is found in the acidic cleft of Vps75, but is not the one involved in histone binding [54]. This region corresponds to the stretch ${ }^{209} \mathrm{KDD}^{211}$ of SET/TAF-I $\beta$ which maps beyond the part we identified as mainly responsible for the interaction with H3. Overall, it appears that SET/TAF-I $\beta$, NAP-1 and Vps75, although structurally very similar, are functionally distinct members of the NAP family of histone chaperones.

\section{Conclusion}

The quantitative study of the histone-binding properties of SET/TAF-I $\beta$ described here and its dissection in structural/ functional domains were based on a type of analysis, that has not been extensively used in the chromatin field, but has allowed us to draw several important conclusions regarding its histone chaperone function. In short, our data showed that SET/TAF-I $\beta$ binds preferentially to histone H3, mainly through its central region, while the dimerisation domain and the acidic tail, both hallmarks of histone chaperones, are not essential for the interaction. On the other hand, binding of H3 to SET/TAF-I $\beta$, is mediated by its core region and does not seem to depend on the presence or the modification status of the $\mathrm{H} 3$ amino-terminal tail. Our findings regarding the ability of distinct SET/TAF-I $\beta$ domains to bind histones are in good agreement (i) with inferences made by close inspection of the tertiary structure, (ii) with the previously deduced 1:1 stoichiometry for the SET/H3 interaction, (iii) with recent suggestions on the relevance of the central $\beta$-sheet motif for the common function of histone chaperones [12] and (iv) with the importance of affinity differences for histone chaperone activity $[45,48]$. Differences of SET/TAF-I $\beta$ with other histone chaperones, that could account for a distinct function, include the clear preference for $\mathrm{H} 3$ binding, the irrelevance of the $\mathrm{H} 3$ tails or their modification status for the interaction and the inability to bind linker histone $\mathrm{H} 1$, together with the recently reported lack of $\mathrm{H} 2 \mathrm{~A} / \mathrm{H} 2 \mathrm{~B}$ dimer stripping activity $[49,50]$.

\section{Methods}

\section{Expression and purification of recombinant proteins}

SET/TAF-I $\beta$ and fragments of SET/TAF-I $\beta$ were subcloned into pGEX vectors (Pharmacia) in frame with glutathione
S-transferase (GST). GST-proteins were expressed in BL21 cells and purified from lysates according to standard procedures [57]. To remove the GST moiety, GST-proteins were immobilized on glutathione beads and digested with the PreScission Protease (Amersham Pharmacia Biotech), 2 units of enzyme $/ 100 \mu \mathrm{g}$ of bound GST-protien in $20 \mu \mathrm{l}$ cleavage buffer ( $50 \mathrm{mM}$ Tris- $\mathrm{HCl}, \mathrm{pH} 7.0,150 \mathrm{mM}$ $\mathrm{NaCl}, 1 \mathrm{mM}$ EDTA, $1 \mathrm{mM}$ DDT, $0.1 \% \mathrm{NP} 40$ ) at $4^{\circ} \mathrm{C}$ for $12 \mathrm{~h}$. Linker histone $\mathrm{H} 1$ and core histones H2A, H2B, H3 and $\mathrm{H} 4$ were obtained from Roche. A pET3a expression vector carrying the cDNAs encoding $\mathrm{H} 3$ and histone $\mathrm{H} 3$ globular domain (aa 27-135) from Xenopus laevis was a kind gift from K. Luger, University of Colorado (California, USA). Recombinant $\mathrm{H} 3$ and tailless $\mathrm{H} 3$ were expressed in E. coli BL21 (DE3) cells and purified under denaturing conditions using SP-sepharose chromatography, as previously described [58]. After removal of urea with extensive dialysis, the samples were lyophilized, dissolved in water and their concentration was adjusted to 1 $\mathrm{mg} / \mathrm{ml}$. The yeast $\mathrm{H} 3$ tail region (1-46) was expressed as a fusion protein with a $\mathrm{N}$-terminal GST tag from a pGET2T expression vector kindly provided from M. Grunstein, University of California and was purified according to standard procedures [59]. Pull-down assays were performed with fresh preparations of all histone constructs.

\section{H3/H4 tetramer assembly}

H3/H4 tetramer reconstitution was based on Luger et al, 1997 [1], with slight modifications. Native H4 was purchased from Roche and dissolved in water at a concentration of $1 \mathrm{mg} / \mathrm{ml}$. Recombinant $\mathrm{H} 3$ was prepared as described above. The two proteins were mixed at equimolar ratio and dialyzed against unfolding buffer ( $6 \mathrm{M}$ Urea, $20 \mathrm{mM}$ Tris-HCl pH 7.5, $5 \mathrm{mM} \beta$-mercaptoethanol) overnight. Tetramer assembly was achieved using the salt-dialysis method as follows: The histone mixture was dialyzed against at least three changes of refolding buffer $(2 \mathrm{M}$ $\mathrm{NaCl}, 10 \mathrm{mM}$ Tris-HCl pH 7.5, 1 mM EDTA, $5 \mathrm{mM} \beta$-mercaptoethanol). Then, the concentration of $\mathrm{NaCl}$ was adjusted by dialysis to $1 \mathrm{M}, 0.5 \mathrm{M}, 0.15 \mathrm{M}$ for $5 \mathrm{~h}$, except the last dialysis step which was left overnight. Precipitated material was removed by centrifugation and the reconstituted tetramer was tested directly in pull-down assays.

\section{GST-pull down assays}

Fusion proteins (approximately 2-3 $\mu$ g each) were immobilized on glutathione-Sepharose beads and incubated with $4 \mu \mathrm{g}$ of histones $(\mathrm{H} 1, \mathrm{H} 2 \mathrm{~A}, \mathrm{H} 2 \mathrm{~B}, \mathrm{H} 3, \mathrm{H} 4)$ in TNMT buffer $(20 \mathrm{mM}$ Tris- $\mathrm{HCl}, \mathrm{pH} 7.5,0.5 \mathrm{M} \mathrm{NaCl}, 2 \mathrm{mM}$ $\mathrm{MgCl}_{2}$ and $1 \%$ Triton X-100) in a total volume of $0.25 \mathrm{ml}$. Reactions were carried out for $60 \mathrm{~min}$ at room temperature. The beads were harvested, washed three times and resuspended in $25 \mu \mathrm{l}$ of SDS sample buffer. For pull-down assays using $(\mathrm{H} 3 / \mathrm{H} 4)_{2}$ tetramers, GST-SET/TAF-I $\beta$ and GST were immobilized on glutathione-agarose beads in the washing buffer $(20 \mathrm{mM}$ Tris- $\mathrm{HCl} \mathrm{pH} 7.5,150 \mathrm{mM}$ 
$\mathrm{NaCl}, 5 \%$ sucrose, $0.1 \% \mathrm{NP}-40,1 \mathrm{mM}$ EDTA, $1 \mathrm{mM}$ DTT, $1 \mathrm{mM}$ PMSF). After washing the beads twice, H3/H4 tetramer was added and incubated for $1 \mathrm{~h}$ in the assay buffer $(20 \mathrm{mM}$ Tris-HCl pH 7.5, $300 \mathrm{mM} \mathrm{NaCl}, 5 \%$ sucrose, 0.1\% NP-40, $1 \mathrm{mM}$ EDTA, $1 \mathrm{mM}$ DTT, $1 \mathrm{mM}$ PMSF, at room temperature. The beads were washed five times with the assay buffer and once with the washing buffer. Bound proteins were eluted with hot SDS-sample buffer and samples were analysed on 13\% SDS-polyacrylamide gels and detected by Coomassie Blue staining.

\section{Fluorescence Spectroscopy Binding Assays}

Binding of the various SET/TAF-I $\beta$ constructs with histones was followed by monitoring the intensity of the fluorescence emitted by the tryptophan residues of SET/TAFI $\beta$ polypeptides upon addition of increasing amounts of calf thymus histone H3 [46,47]. A Hitachi F-2500 fluorescence spectrophotometer, fitted with a thermostatically controlled jacketed cell holder and interfaced with a Neslab RTE-111 water-bath was used. Fluorescence emission spectra in the range 300-400 $\mathrm{nm}$ were recorded with an excitation wavelength of $295 \mathrm{~nm}$. SET/TAF-I $\beta$ polypeptides at several initial concentrations ranging from 0.2 to $4 \mu \mathrm{M}$ were titrated with increasing amounts of purified calf thymus $\mathrm{H} 3$ stock solution in PBS buffer. All measurements were performed at $25^{\circ} \mathrm{C}$ in a quartz cell with path length of $1 \mathrm{~cm}$ (Hellma) under constant stirring. After each ligand addition, the samples were left to equilibrate for $10 \mathrm{~min}$ before the equilibrium fluorescence readings were recorded. The dissociation constant $\left(K_{d}\right)$ for the complexes formed was estimated by non-linear regression analysis (KaleidaGraph, Synergy Software) relating the change in SET fluorescence intensity at $345 \mathrm{~nm}$ (corrected for buffer contribution and for dilution) to the total added histone concentration through Equation 1:

$$
F=\left(F_{0}+F_{\max }[L] / K_{d}\right) /\left(1+[L] / K_{d}\right)
$$

where $\mathrm{F}, F_{0}$, and $F_{\max }$ are the fluorescence intensities measured at each titration point, at zero ligand concentration and at saturation, respectively, and $[L]$ is the concentration of free ligand calculated by solving the quadratic equation

$$
[L]^{2}+[L]\left([P]_{0}-[L]_{0}+K_{d}\right)-K_{d}[L]_{0}=0
$$

where $[\mathrm{P}]_{0}$ and $[L]_{0}$ are the protein and total ligand concentrations added, respectively. Each titration was repeated three times.

\section{Circular Dichroism}

Circular dichroism spectra in the Far UV range (180-260 $\mathrm{nm}$ ) were recorded on a Jasco J-815 spectropolarimeter interfaced with a Peltier element for temperature control. The instrument was calibrated with a $0.1 \%$ aqueous solu- tion of $d$-10-camphor sulfonic acid. Stock solutions of SET/TAF-IB polypeptides were in $50 \mathrm{mM}$ Tris- $\mathrm{HCl} \mathrm{pH} 7.5$, $150 \mathrm{mM} \mathrm{NaCl}, 0.5 \mathrm{mM}$ DTT, $1 \mathrm{mM}$ EDTA and their concentration ranged from 10 to $75 \mu \mathrm{M}$. Spectra were recorded at $25^{\circ} \mathrm{C}$, with $0.2 \mathrm{~nm}$ resolution, averaged over five scans and were baseline corrected by subtraction of the buffer spectrum at the same temperature. Quartz cells with path lengths of 0.02 or $0.1 \mathrm{~cm}$ were used (Hellma). The combined absorbance of cell, sample and solvent was kept at $<1$ over the measured range. Secondary structure was estimated as described previously [60]. Molecular images were generated with PyMol [61].

\section{Authors' contributions}

$\mathrm{ZK}$, AE carried out cloning, purification of wild-type and mutant proteins and performed fluorescence spectroscopy-based binding assays. IS, SL carried out the tetramer assembly, purification of wild-type and mutant proteins and GST-pull down assays. EN participated in the design and coordination of the study. AP and TP designed the study, supervised data collection and analysis and drafted the manuscript. All authors read and approved the final manuscript.

\section{Acknowledgements}

The research Project was co-funded by the European Union - European Social Fund (ESF) \& National Sources, in the framework of the program "Pythagoras II" of the "Operational Program for Education and Initial Vocational Training" of the $3^{\text {rd }}$ Community Support Framework of the Hellenic Ministry of Education. We would like to thank K. Luger (University of Colorado) for $\mathrm{H} 3$ plasmids and M. Grunstein (University of California) for the H3-tail plasmid.

\section{References}

I. Luger K, Mader AW, Richmond RK, Sargent DF, Richmond TJ: Crystal structure of the nucleosome core particle at $2.8 \mathrm{~A}$ resolution. Nature 1997, 389:25I-260.

2. Horn PJ, Peterson CL: Molecular biology. Chromatin higher order folding-wrapping up transcription. Science 2002, 297: | $824-1827$

3. Mellor J: The dynamics of chromatin remodeling at promoters. Mol Cell 2005, I 9:|47-I57.

4. Varga-Weisz PD, Becker PB: Regulation of higher-order chromatin structures by nucleosome-remodelling factors. Curr Opin Genet Dev 2006, 16:151-156.

5. Langst G, Becker PB: Nucleosome remodeling: one mechanism, many phenomena? Biochim Biophys Acta 2004, I 677:58-63.

6. Cosgrove MS: Histone proteomics and the epigenetic regulation of nucleosome mobility. Expert Rev Proteomics 2007, 4:465-478.

7. Strahl BD, Allis CD: The language of covalent histone modifications. Nature 2000, 403:4I-45.

8. Cosgrove MS, Boeke JD, Wolberger C: Regulated nucleosome mobility and the histone code. Nat Struct Mol Biol 2004, I I : 1037-1043.

9. Tyler JK: Chromatin assembly. Cooperation between histone chaperones and ATP-dependent nucleosome remodeling machines. Eur J Biochem 2002, 269:2268-2274.

10. Loyola A, Almouzni G: Histone chaperones, a supporting role in the limelight. Biochim Biophys Acta 2004, I 677:3- II.

II. Park Y], Luger K: Histone chaperones in nucleosome eviction and histone exchange. Curr Opin Struct Biol 2008, I 8:282-289.

12. Park YJ, Luger K: The structure of nucleosome assembly protein I. Proc Natl Acad Sci USA 2006, I 03:1248-I 253. 
13. Park Y], Luger K: Structure and function of nucleosome assembly proteins. Biochem Cell Biol 2006, 84:549-558.

14. Smith S, Stillman B: Purification and characterization of CAF-I, a human cell factor required for chromatin assembly during DNA replication in vitro. Cell 1989, 58:15-25.

15. Kleinschmidt JA, Dingwall C, Maier G, Franke WW: Molecular characterization of a karyophilic, histone-binding protein: cDNA cloning, amino acid sequence and expression of nuclear protein NI/N2 of Xenopus laevis. EMBO J 1986, 5:3547-3552.

16. Laskey RA, Honda BM, Mills AD, Finch JT: Nucleosomes are assembled by an acidic protein which binds histones and transfers them to DNA. Nature 1978, 275:416-420.

17. English CM, Adkins MW, Carson JJ, Churchill ME, Tyler JK: Structural basis for the histone chaperone activity of Asfl. Cell 2006, I 27:495-508.

18. Ray-Gallet D, Quivy JP, Scamps C, Martini EM, Lipinski M, Almouzni G: HIRA is critical for a nucleosome assembly pathway independent of DNA synthesis. Mol Cell 2002, 9:1091-I 100.

19. von Lindern M, van Baal S, Wiegant J, Raap A, Hagemeijer A, Grosveld G: Can, a putative oncogene associated with myeloid leukemogenesis, may be activated by fusion of its 3 ' half to different genes: characterization of the set gene. Mol Cell Biol I992, I 2:3346-3355

20. Adachi $Y$, Pavlakis GN, Copeland TD: Identification and characterization of SET, a nuclear phosphoprotein encoded by the translocation break point in acute undifferentiated leukemia. J Biol Chem 1994, 269:2258-2262.

21. Nagata K, Kawase H, Handa H, Yano K, Yamasaki M, Ishimi Y, Okuda A, Kikuchi A, Matsumoto K: Replication factor encoded by a putative oncogene, set, associated with myeloid leukemogenesis. Proc Natl Acad Sci USA 1995, 92:4279-4283.

22. Nagata K, Saito S, Okuwaki M, Kawase H, Furuya A, Kusano A, Hanai $\mathrm{N}$, Okuda $\mathrm{A}$, Kikuchi $\mathrm{A}$ : Cellular localization and expression of template-activating factor I in different cell types. Exp Cell Res 1998, 240:274-28I.

23. Estanyol JM, Jaumot M, Casanovas O, Rodriguez-Vilarrupla A, Agell N, Bachs O: The protein SET regulates the inhibitory effect of p2 I(Cip I) on cyclin E-cyclin-dependent kinase 2 activity. Biol Chem 1999, 274:3316I-33I65.

24. Canela N, Rodriguez-Vilarrupla A, Estanyol JM, Diaz C, Pujol MJ, Agell $\mathrm{N}$, Bachs O: The SET protein regulates G2/M transition by modulating cyclin B-cyclin-dependent kinase I activity. J Biol Chem 2003, 278: I I58-1164

25. Ou D, Li Q, Lim HY, Cheung NS, Li R, Wang JH, Qi RZ: The protein SET binds the neuronal Cdk5 activator p35nck5a and modulates Cdk5/p35nck5a activity. J Biol Chem 2002, 277:7324-7332.

26. Fan Z, Beresford PJ, Oh DY, Zhang D, Lieberman J: Tumor Suppressor NM23-HI Is a Granzyme A-Activated DNase during CTL-Mediated Apoptosis, and the Nucleosome Assembly Protein SET Is Its Inhibitor. Cell 2003, I I 2:659-672.

27. Matsumoto K, Nagata K, Ui M, Hanaoka F: Template activating factor $I$, a novel host factor required to stimulate the adenovirus core DNA replication. J Biol Chem 1993, 268: I0582-I 0587.

28. Matsumoto K, Okuwaki M, Kawase H, Handa H, Hanaoka F, Nagata $\mathrm{K}$ : Stimulation of DNA transcription by the replication factor from the adenovirus genome in a chromatin-like structure. J Biol Chem 1995, 270:9645-9650.

29. Pegoraro G, Marcello A, Myers MP, Giacca M: Regulation of adeno-associated virus DNA replication by the cellular TAFI/set complex. J Virol 2006, 80:6855-6864.

30. Haruki H, Gyurcsik B, Okuwaki M, Nagata K: Ternary complex formation between DNA-adenovirus core protein VII and TAF-lbeta/SET, an acidic molecular chaperone. FEBS Lett 2003, 555:52।-527.

31. Matsumoto K, Nagata K, Miyaji-Yamaguchi M, Kikuchi A, Tsujimoto M: Sperm chromatin decondensation by template activating factor I through direct interaction with basic proteins. Mol Cell Biol 1999, 19:6940-6952.

32. Matsumoto K, Nagata K, Okuwaki M, Tsujimoto M: Histone- and chromatin-binding activity of template activating factor-I. FEBS Lett 1999, 463:285-288.

33. Kutney $S N$, Hong R, Macfarlan T, Chakravarti D: A signaling role of histone-binding proteins and INHAT subunits pp32 and Set/ TAF-lbeta in integrating chromatin hypoacetylation and transcriptional repression. J Biol Chem 2004, 279:30850-30855.
34. Schneider R, Bannister AJ, Weise C, Kouzarides T: Direct binding of INHAT to $\mathbf{H} 3$ tails disrupted by modifications. J Biol Chem 2004, 279:23859-23862.

35. Seo SB, McNamara P, Heo S, Turner A, Lane WS, Chakravarti D Regulation of histone acetylation and transcription by INHAT, a human cellular complex containing the set oncoprotein. Cell 200I, 104:1 I9-130.

36. Karetsou Z, Martic G, Tavoulari S, Christoforidis S, Wilm M, Gruss C, Papamarcaki T: Prothymosin alpha associates with the oncoprotein SET and is involved in chromatin decondensation. FEBS Lett 2004, 577:496-500.

37. Karetsou Z, Martic G, Sflomos G, Papamarcaki T: The histone chaperone SET/TAF-lbeta interacts functionally with the CREB-binding protein. Biochem Biophys Res Commun 2005, 335:322-327.

38. Kato K, Miyaji-Yamaguchi M, Okuwaki M, Nagata K: Histone acetylation-independent transcription stimulation by a histone chaperone. Nucleic Acids Res 2007, 35:705-7I5.

39. Ichijo T, Chrousos GP, Kino T: Activated glucocorticoid receptor interacts with the INHAT component Set/TAF-Ibeta and releases it from a glucocorticoid-responsive gene promoter, relieving repression: implications for the pathogenesis of glucocorticoid resistance in acute undifferentiated leukemia with Set-Can translocation. Mol Cel Endocrinol 2008, 283:|9-3I.

40. Miyaji-Yamaguchi M, Okuwaki M, Nagata K: Coiled-coil structuremediated dimerization of template activating factor-I is critical for its chromatin remodeling activity. J Mol Biol 1999, 290:547-557.

4I. McBryant SJ, Park YJ, Abernathy SM, Laybourn PJ, Nyborg JK, Luger $\mathrm{K}$ : Preferential binding of the histone $(\mathrm{H} 3-\mathrm{H} 4) 2$ tetramer by NAPI is mediated by the amino-terminal histone tails. J Biol Chem 2003, 278:44574-44583.

42. Okuwaki M, Nagata K: Template activating factor-Iremodels the chromatin structure and stimulates transcription fromthe chromatin template. J Biol Chem 1998, 273:345||-345|8.

43. De Koning L, Corpet A, Haber JE, Almouzni G: Histone chaperones: an escort network regulating histone traffic. Nat Struct Mol Biol 2007, 14:997-1007.

44. Eitoku M, Sato L, Senda T, Horikoshi M: Histone chaperones: $\mathbf{3 0}$ years from isolation to elucidation of the mechanisms of nucleosome assembly and disassembly. Cell Mol Life Sci 2008, 65:414-444

45. Mazurkiewicz J, Kepert JF, Rippe K: On the mechanism of nucleosome assembly by histone chaperone NAPI. I Biol Chem 2006, 28 I: 16462-16472

46. Politou AS, Spadaccini R, Joseph C, Brannetti B, Guerrini R, HelmerCitterich M, Salvadori S, Temussi PA, Pastore A: The SH3 domain of nebulin binds selectively to type II peptides: theoretical prediction and experimental validation. J Mol Biol 2002, 3 16:305-3I5.

47. Viguera AR, Arrondo JL, Musacchio A, Saraste M, Serrano L: Characterization of the interaction of natural proline-rich peptides with five different SH3 domains. Biochemistry 1994, 33:10925-10933.

48. Kepert JF, Mazurkiewicz J, Heuvelman GL, Toth KF, Rippe K: NAP I modulates binding of linker histone $\mathrm{HI}$ to chromatin and induces an extended chromatin fiber conformation. J Biol Chem 2005, 280:34063-34072

49. Okuwaki M, Kato K, Shimahara H, Tate S, Nagata K: Assembly and disassembly of nucleosome core particles containing histone variants by human nucleosome assembly protein I. Mol Cell Biol 2005, 25: 10639-1065।.

50. Park YJ, Chodaparambil JV, Bao Y, McBryant SJ, Luger K: Nucleosome assembly protein I exchanges histone H2A-H2B dimers and assists nucleosome sliding. I Biol Chem 2005, 280: $1817-1825$

5I. Muto S, Senda M, Akai Y, Sato L, Suzuki T, Nagai R, Senda T, Horikoshi M: Relationship between the structure of SET/TAF-lbeta/ INHAT and its histone chaperone activity. Proc Natl Acad Sci USA 2007, 104:4285-4290.

52. English CM, Maluf NK, Tripet B, Churchill ME, Tyler JK: ASFI binds to a heterodimer of histones $\mathrm{H} 3$ and $\mathrm{H} 4$ : a two-step mechanism for the assembly of the $\mathrm{H} 3-\mathrm{H} 4$ heterotetramer on DNA. Biochemistry 2005, 44: |3673-13682. 
53. Selth L, Svejstrup JQ: Vps75, a new yeast member of the NAP histone chaperone family. J Biol Chem 2007, 282:I2358-1 2362.

54. Berndsen CE, Tsubota T, Lindner SE, Lee S, Holton JM, Kaufman PD Keck JL, Denu JM: Molecular functions of the histone acetyltransferase chaperone complex Rtt I09-Vps75. Nat Struct Mol Biol 2008, 15:948-956.

55. Park Y], Sudhoff KB, Andrews AJ, Stargell LA, Luger K: Histone chaperone specificity in Rtt 109 activation. Nat Struct Mol Biol 2008, I 5:957-964.

56. Tang Y, Meeth K, Jiang E, Luo C, Marmorstein R: Structure of Vps75 and implications for histone chaperone function. Proc Natl Acad Sci USA 2008, 105: 12206-122II.

57. Sambrook J, Fritsch EF, Maniatis T: Molecular Cloning: A Laboratory Manual Cold Spring Harbor, Cold Spring Harbor Press; 1989.

58. Luger K, Rechsteiner TJ, Flaus AJ, Waye MM, Richmond TJ: Characterization of nucleosome core particles containing histone proteins made in bacteria. J Mol Biol 1997, 272:30I-3II.

59. Ling X, Harkness TA, Schultz MC, Fisher-Adams G, Grunstein M: Yeast histone $\mathrm{H} 3$ and $\mathrm{H} 4$ amino termini are important for nucleosome assembly in vivo and in vitro: redundant and position-independent functions in assembly but not in gene regulation. Genes Dev 1996, 10:686-699.

60. Provencher SW, Glockner J: Estimation of globular protein secondary structure from circular dichroism. Biochemistry 1981, 20:33-37.

61. Delano WL: The Pymol MolecularGraphics System. Delano Scientific: San Carlos, CA; 2002

Publish with Biomed Central and every scientist can read your work free of charge

"BioMed Central will be the most significant development for disseminating the results of biomedical research in our lifetime. "

Sir Paul Nurse, Cancer Research UK

Your research papers will be:

- available free of charge to the entire biomedical community

- peer reviewed and published immediately upon acceptance

- cited in PubMed and archived on PubMed Central

- yours - you keep the copyright
BioMedcentral 